\title{
A time-synchronous domain decomposition code for multiphysics systems
}

\author{
M. G. Trefry* J. Öhman ${ }^{\dagger} \quad$ D. S. Whyte $\quad$ G. B. Davis* \\ (Received 7 August 2000)
}

*CSIRO Land and Water, AustraliA. mailto:mike.trefry@per.clw.csiro.au and mailto:greg.davis@per.clw.csiro.au

${ }^{\dagger}$ School of Engineering, University of Uppsala, SwEDEN. mailto: johanoman@hotmail.com

${ }^{\ddagger}$ Department of Chemical Engineering, University of Melbourne, Australia. mailto:d.whyte@chemeng . unimelb.edu . au

${ }^{0}$ See http: //anziamj.austms .org.au/V42/CTAC99/Tref for this article and ancillary services, (C) Austral. Mathematical Soc. 2000. Published 27 Nov 2000. 


\section{Abstract}

In assessing risks of exposure to toxic contaminants in environmental systems, the transport dynamics of contaminants through complex heterogeneous media is a key determinant. Quantitative predictions of contaminant concentrations and fluxes are often required for systems involving different classes of physical dynamics and sharply discontinuous material properties. For many volatile contaminants, special conservation laws apply at interfaces between neighbouring system subdomains. These conservation laws couple dependent variables between the subdomains in a dynamic sense. Within the subdomains, essentially arbitrary physical processes may occur, possibly coupling many dependent variables.

We describe the development of a code applicable to such multiphysics problems. The code uses an advanced black box solver as an engine to solve the partial differential equations appropriate to each subdomain. Simple geometric domain decomposition is used to define subdomains. In this first implementation, parallel instances of the solver engine (that is, in each subdomain) communicate synchronously with each other to facilitate time-stepping of the total system solution. The interfacial conservation laws act as constraints to the subdomain solutions, effectively providing dynamic internal boundary conditions. Convergence characteristics of the discretised interface algorithms are discussed in terms of validations against recently derived analytical solutions for diffusion-limited transport in partitioning laminates. Finally, an application of the code to coupled benzene vapour transport in variably saturated porous and permeable media is outlined. 


\section{Contents}

1 Introduction

C1445

2 Numerical implementation-discretisation and synchronisation

C1450

3 Convergence behaviour

C1453

4 Application-hydrocarbon biodegradation in a capped soil column

C1458

5 Conclusions

C1462

References

C1462

\section{Introduction}

Risk assessment methodologies pertinent to hydrocarbon exposure pathways rely upon accurate models of hydrocarbon transport in target environmental systems. Many fundamental principles governing hydrocarbon vapour phase and non-aqueous phase liquid (NAPL) transport are reasonably well understood, although the accurate description of hydrocarbon transport dynamics in multiphase and/or strongly heterogeneous systems still presents difficulty 
to the theoretician. The situation can be exacerbated in risk assessment studies because exposure pathways typically involve the transport/migration of target contaminants through several different physical phases and media. For example, domestic vapour inhalation risks may arise from solvent migration from an advecting dissolved phase in soil material or groundwater, subsequent diffusion through a variably saturated porous phase, before release into a confined, semi-confined, or unconfined atmospheric phase. Tools offering quantitative solutions for such complex problems need to cater for diverse dynamical equation systems, strong heterogeneity or structure in material definitions, and the possibility of several differing characteristic time scales. Furthermore, the possibility of a diverse range of conservation laws applying at interfaces between system phases must be admitted, and is of particular interest in this work.

Attention is now directed to complex systems of mixed physical processes and mixed, non-overlapping media. That is, the overall system domain may consist of several distinct media types, in an arbitrary arrangement and multiplicity. Also, within the system domain, several different physical processes may apply. Some of these may extend over several, or all, media, and some may be confined to single media. Other processes may be coupled within media, or across media. It is assumed that the geometric statement of the media arrangement is static, and that the physical processes may be modelled in terms of sets of differential operators, which have as domains subsets of the total system domain (see Figure 1). In a domain decomposition approach to the overall problem, solution may be facilitated by dividing the complex system into simpler subproblems. In many studies this decomposition is per- 
formed on geometric grounds, exploiting simplifications due to symmetry in boundary conditions or in the coefficients of the differential operators. In the present case, where different differential operators, $H^{m}$, may apply in different regions of the system (indexed by $m$ ), the decomposition can take into account not only boundary conditions and operator coefficients, but also the various forms of the local physical operators. Geometric domain decomposition therefore results in subdomains, which may or may not be spatially congruent with the media comprising the system.

Clearly, the identification of the operators $H^{m}$ is problem-dependent. In the context of contaminant transport in porous/permeable systems, each operator $H^{m}$ is typically represented by a coupled set of parabolic equations with a variety of source/sink terms, which may be linear (exponential decay, volatilisation) or non-linear (isothermal sorption, Monod kinetic). Including the possibility of fluid flow may require Navier-Stokes operators, while coupled heat and reaction chemistry may lead to mechanical deformations of the subdomains requiring stress tensor formulations, and so on. In this work, attention is restricted to operators able to be expressed in terms of coupled sets of partial differential equations that are, at most, first order in time and second order in space. This, for example, includes the standard continuum transport models used in soil contamination studies [1].

Whilst interfacial conditions appropriate for classical diffusion between static media are well understood, yielding continuous (but not necessarily differentiable) concentrations, not all physical dependent variables obey these types of continuity relations. More problematic interface conditions are pre- 


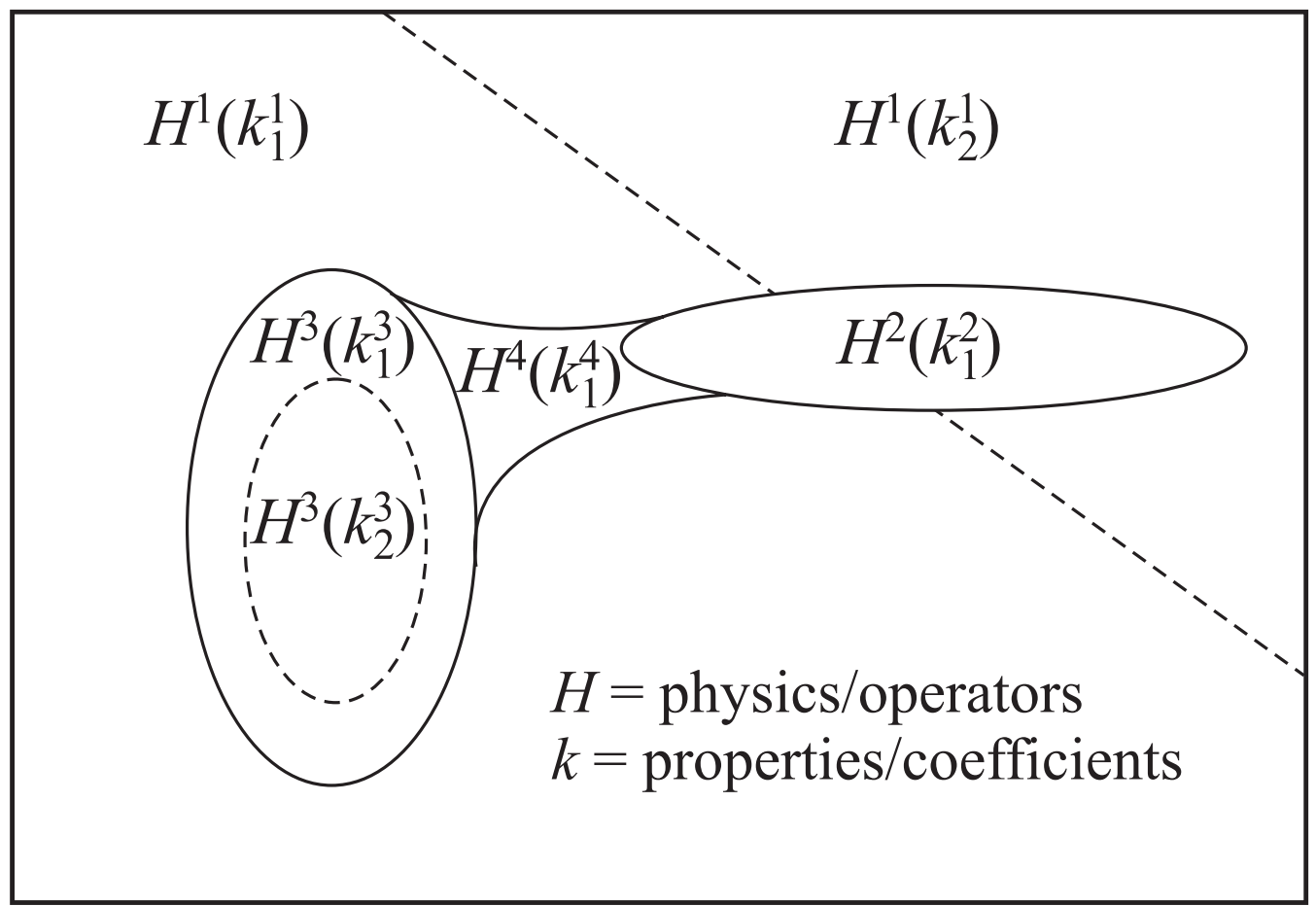

Figure 1: A multiphysics system. Subdomains with different physical processes (differential operators) $H^{m}$ are separated by interfaces (solid lines). Dashed lines indicate changes in subdomain properties (operator coefficients) $k_{i}^{m}$. Boundary conditions are specified along the bounding box. 
sented by systems involving chemical partitioning interactions, which can lead to sharp discontinuities in concentrations across physical phase interfaces. Continuum approaches provide valuable simplifications for transport within porous media with microscopic structure [2], however for the present case explicit solutions are required for the coupled transport and interface equations.

Figure 1 depicts a system where media properties and physical dynamics are discontinuous at a macroscale. For such systems, continuum models must be enhanced to incorporate conservation laws appropriate to the subdomain interfaces; these interfacial laws are critical drivers in the system. Recent work on the transport of volatile species in mixed media [3] has identified two simple classes of interfacial conditions. Consider an interface $\Gamma^{m, n}$ between subdomains $m$ and $n$ characterised by physical processes $H^{m}$ and $H^{n}$. Dependent variable $u_{i}^{m}$ (the $i$-th dependent variable in subdomain $m$ ) may be coupled with $u_{j}^{n}$ across the interface $\Gamma^{m, n}$ according to either a local equilibrium law:

\section{Equilibrium Condition}

$$
u_{i}^{m}\left(\underline{x}_{k}, t\right)=\alpha_{i, j}^{m, n} u_{j}^{n}\left(\underline{x}_{k}, t\right) \quad \forall \underline{x}_{k} \in \Gamma^{m, n}, t>0
$$

or by a resistive law relating the interfacial fluxes, $q$ (which are proportional to gradients of the $u$ variables): 


\section{Rate-Limited Condition}

$$
\left.\begin{array}{c}
q_{i}^{m}\left(\underline{x}_{k}, t\right)=q_{j}^{n}\left(\underline{x}_{k}, t\right)=P_{i, j}^{m, n}\left(\underline{x}_{k}, t\right) \\
P_{i, j}^{m, n}\left(\underline{x}_{k}, t\right)=\beta_{i, j}^{m, n}\left(u_{i}^{m}\left(\underline{x}_{k}, t\right)-\alpha_{i, j}^{m, n} u_{j}^{n}\left(\underline{x}_{k}, t\right)\right)
\end{array}\right\} \forall \underline{x}_{k} \in \Gamma^{m, n}, t>0
$$

The non-negative parameter $\alpha$ is referred to as a partitioning coefficient, and can range over many orders of magnitude for various combinations of chemical compounds and transport media. At equilibrium, both interfacial conditions yield the same discontinuous distribution of independent variables across the interface, determined by $\alpha$; however at intermediate times the ratelimited condition generates disequilibria through the form of the interface "potential" $P$. Here the rate of relaxation to the partitioning equilibrium is governed by the non-negative mass transfer coefficient $\beta$.

\section{Numerical implementation-discretisation and synchronisation}

For the class of pde systems discussed above, reliable "black box" solvers are readily available. In a finite difference context, the VLUGR software package [4] provides a flexible and stable solver engine for handling the internal dynamics of each subdomain. In two dimensions, VLUGR solves the nonlinear system

$$
H\left(t, x, y, u, u_{t}, u_{x}, u_{y}, u_{x y}, u_{x x}, u_{y y}\right)=0
$$


subject to the boundary and initial conditions

$$
B\left(t, x, y, u, u_{x}, u_{y}\right)=0 \quad \text { and } \quad u\left(x, y, t_{0}\right)=u_{0}(x, y)
$$

using a local grid refinement technique. Here, as in the discussion above, the solution $u=\left(u_{1}, u_{2}, \ldots, u_{L}\right)$ is a vector quantity of finite dimension $L$. VLUGR incorporates an automatic time stepping procedure, with time steps dynamically adjusted so that the solution error remains within user-input bounds.

In the context of multiphysics processes, parallel instances of the VLUGR solver are assigned to the subdomains identified in the geometric decomposition of the overall problem. It remains to establish a time stepping implementation of the interfacial conditions linking the dynamics in the subdomains. Discussions on time stepping techniques for domain decomposition problems have been presented by Mathew et al. [5] and Amitai et al. [6]. Here, as a first implementation, a simple static-synchronous approach is used (see Table 1). For clarity the method is outlined in one dimension, although a two-dimensional implementation has already been completed. Consider a problem domain represented by the interval $x \in\left[x_{\min }, x_{\max }\right]$, split into two subdomains contiguous at the interface $x_{k}$ associated with problem operators $H^{1}$ and $H^{2}$. Coordinate arrays in the subdomains are determined by the discretisations $\Delta x_{1}$ and $\Delta x_{2}$. At system time $t$ the subdomain solutions are represented by

$$
\text { and } \begin{gathered}
\left\{u_{i}^{1}\left(x_{\min }, t\right), u_{i}^{1}\left(x_{\min }+\Delta x_{1}, t\right), \ldots, u_{i}^{1}\left(x_{k}, t\right)\right\} \\
\left\{u_{j}^{2}\left(x_{k}, t\right), u_{j}^{2}\left(x_{k}+\Delta x_{2}, t\right), \ldots, u_{j}^{2}\left(x_{\max }, t\right)\right\}
\end{gathered}
$$


System boundary conditions apply at $x_{\min }$ and $x_{\max }$. For an equilibrium interface condition (equation (1)) at $x_{k}$, relating solution components $u_{i}^{1}$ and $u_{j}^{2}$, the solutions can be advanced to system time $t+\Delta t^{\text {system }}$, where $\Delta t^{\text {system }}$ is a (static) constant time step input by the user, by solving $H^{1}$ and $H^{2}$ in turn, subject to zero gradient conditions at $x_{k}$. The equilibrium condition is imposed at the end of each system time step by modulating the local grid point values according to equation (1). For example, at the completion of the system time step leading to system time $t$, and neglecting volumetric effects arising from variable porosities, a mass-balanced partitioning condition leads to the updated values (denoted by ${ }^{*}$ )

$$
u_{i}^{1 *}\left(x_{k}, t\right)=\frac{\alpha_{i, j}^{1,2} M_{i, j}^{1,2}}{\alpha_{i, j}^{1,2} \Delta x_{1}+\Delta x_{2}}=\alpha_{i, j}^{1,2} u_{j}^{2 *}\left(x_{k}, t\right)
$$

where $M_{i, j}^{1,2}$ is the integrated mass in solution components $i$ and $j$ local to the interface. $M$ is approximated using a simple trapezoidal rule as follows.

$$
\begin{array}{r}
M_{i, j}^{1,2}(t) \approx \frac{\Delta x_{1}}{2}\left(u_{i}^{1}\left(x_{k}-\Delta x_{1}, t\right)+u_{i}^{1}\left(x_{k}, t\right)\right)+ \\
\frac{\Delta x_{2}}{2}\left(u_{j}^{2}\left(x_{k}, t\right)+u_{j}^{2}\left(x_{k}+\Delta x_{2}, t\right)\right)
\end{array}
$$

This trapezoidal approximation introduces mass balance errors that scale as $\Delta x^{2}$, and can easily be replaced by more accurate schemes if required. Updated values at the extra grid points $u_{i}^{1}\left(x_{k}-\Delta x_{1}, t\right)$ and $u_{j}^{2}\left(x_{k}+\Delta x_{2}, t\right)$ used in determining $M$ follow from the zero gradient assumption and equation (6). These extra points are included to overcome indeterminacies associated with the zero gradient subdomain conditions. $H^{1}$ and $H^{2}$ are then integrated 
for one time step, and the above partitioning scheme is applied, and so on. Thus, solutions in neighbouring subdomains communicate with each other simultaneously at the end of each fixed system time step by a simple iterationfree procedure. For the rate-limited interface conditions, equation (2), the procedure requires the calculation of the $P_{i, j}^{1,2}$ quantity at the end of each system time step, applying the resulting interfacial gradient conditions for the duration of the next system time step. In this case, single points in each domain, $u_{i}^{1}\left(x_{k}, t\right)$ and $u_{j}^{2}\left(x_{k}, t\right)$, are sufficient to fix $P$. In the following sections the convergence properties of these simple interfacial schemes are examined briefly, and an example application is sketched.

TABLE 1: Static-synchronous interfacial time stepping scheme.

user input of $\Delta t^{\text {system }}$

1 advance all operators $H^{i}$ by $\Delta t^{\text {system }}$

$t^{\text {system }}=t^{\text {system }}+\Delta t^{\text {system }}$

updateinterfacial conditions using $H^{i}$ solutions

goto 1

\section{Convergence behaviour}

The interfacial schemes outlined above have been validated against analytical solutions for representative transport processes in laminate media subjected 
to steady and transient system boundary conditions [2, 3, 7, 8]. Here convergence results are presented for numerical solutions for steady boundary condition problems. Convergence is measured with respect to analytical solutions gained by eigenfunction expansion techniques [3]. Table 2 shows results for a one-dimensional system $x \in[0,2]$ composed of two subdomains with equilibrium interface condition located at $x_{\text {int }}=1$. Within each subdomain a scalar diffusion equation applies:

$$
\begin{array}{ll}
\text { Subdomain } 1 & \frac{\partial u_{1}^{1}}{\partial t}=d_{1} \frac{\partial^{2} u_{1}^{1}}{\partial x^{2}} \\
\text { Subdomain } 2 & \frac{\partial u_{1}^{2}}{\partial t}=d_{2} \frac{\partial^{2} u_{1}^{2}}{\partial x^{2}}
\end{array}
$$

where $d_{1}$ and $d_{2}$ are simple diffusion coefficients with values 5 and 0.05 , respectively. Zero-gradient boundary conditions apply at $x=0$ and $x=2$. The initial condition is $u_{1}^{1}(x, 0)=1,0 \leq x \leq x_{\text {int }}$ and $u_{1}^{2}(x, 0)=0, x_{\text {int }} \leq x \leq 1$. RMS percentage errors between numerical and analytical results are calculated by summing over all grid points within 0.1 of the interface in each subdomain. Each entry in Table 2 contains three values, corresponding to $\alpha_{1,1}^{1,2}=1 / 500,1$ and 500, respectively. Table 3 shows corresponding results for the rate-limited interface condition, with $\beta_{1,1}^{1,2}=0.01$ and for two values of partitioning coefficient, $\alpha_{1,1}^{1,2}=1 / 500$ and 500, respectively. Stability problems in the rate-limited interfacial scheme for non-equilibrium systems are common for $\beta$ values greater than unity, due to the large transient gradients applying at the interfaces.

The tables show that uniform convergence behaviour does not apply for all discretisations in the current implementation. In Table 2, for the exhibited 
TABLE 2: Convergence behaviour of the equilibrium interface condition. Subdomains have matching discretisations.

\begin{tabular}{rr|ccc}
\multicolumn{2}{r|}{} & \multicolumn{4}{c}{$\Delta t$} \\
\multicolumn{2}{r|}{ RMS Error (\%) } & 0.001 & 0.0005 & 0.00005 \\
\hline \multirow{2}{*}{$\Delta x$} & 0.02 & $86.0 / 4.53 / 5.97$ & $21.8 / 5.14 / 6.42$ & $3.77 / 5.73 / 6.82$ \\
& 0.01 & $366 / 0.29 / 1.68$ & $98.9 / 1.57 / 2.55$ & $1.90 / 2.77 / 3.34$ \\
& 0.005 & $1172 / 3.94 / 1.71$ & $381 / 1.27 / 0.01$ & $10.0 / 1.18 / 1.54$ \\
\hline
\end{tabular}

TABLE 3: Convergence behaviour of the rate-limited interface condition. Subdomains have matching discretisations. Numbers in parentheses indicate powers of ten.

\begin{tabular}{rr|ccc} 
& \multicolumn{4}{c}{$\Delta t$} \\
\multicolumn{2}{r|r}{ RMS Error (\%) } & 0.001 & 0.0005 & 0.00005 \\
\hline \multirow{2}{*}{$\Delta x$} & 0.02 & $23.7 / 62.4$ & $23.5 / 64.4$ & $23.6 / 67.0$ \\
& 0.01 & $2.33 / 12.7$ & $2.40 / 6.91$ & $2.47 / 0.74$ \\
& 0.005 & $1.09 / 3.2(-3)$ & $1.17 / 8.2(-3)$ & $1.23 / 1.7(-2)$ \\
\hline
\end{tabular}


combinations of $\Delta x$ and $\Delta t$, reasonably accurate solutions were gained for $\alpha_{1,1}^{1,2}=1$ and 500, where departures from the initial conditions are small, but gross errors were encountered for $\alpha_{1,1}^{1,2}=1 / 500$, where finer spatial discretisations led to increased errors. In this case, for relatively coarse discretisations, the RMS errors were found to scale approximately as $(\Delta t / \Delta x)^{2}$. For finer discretisations, the RMS error scaled approximately bilinearly, i.e. as $\Delta x \Delta t$. In Table 3, the convergence picture is again complicated. For decreasing $\Delta x$, the RMS error tended towards first order in nature, while theRMS error was approximately independent of $\Delta t$, except when $\Delta x=0.01$ and $\alpha_{1,1}^{1,2}=500$. Again, best accuracy was achieved for $\alpha_{1,1}^{1,2}=500$, where departures from the initial conditions were the least.

In summary, it is clear from Table 2 that optimal discretisations are unlikely to be found by simple linear programming approaches in $(\Delta x, \Delta t)$ space without first locating the region of approximate linear convergence. There are several reasons for this. First, VLUGR uses an error formulation that is grid dependent. This error norm is used to estimate both time step lengths and solution convergence. Hence, comparing multi-domain results calculated from different spatial and temporal grids is complicated. This may be part of the reason that the results shown in the tables are uneven, even though internal VLUGR error parameters were held constant throughout the simulations. Second, the VLUGR engine uses an adaptive time stepping approach, so that several internal subdomain time steps may be performed in between the interface communication stage indicated by equation (6). Thus subdomain solutions may repeatedly be advanced using fixed interfacial conditions. 
This may be overcome within the current temporal implementation by employing exceedingly fine interface time steps so that the number of internal subdomain time steps between interfacial updates is minimised.

Another approach to overcome this poor interfacial performance is to allow the subdomain dynamics to determine the interfacial time steps. Consider a system with $N$ physical operators $H^{i}$ acting in $N$ subdomains $i$. At a given system time $t^{\text {system }}$, in each subdomain $i$ the solver instance will produce an estimate of the maximum time step $\Delta t_{\max }^{i}$ it may integrate over so that the associated solution error bound will be satisfied. The system time step $\Delta t^{\text {system }}$ may then be chosen to be the minimum of the subdomain time steps. The system (i.e. all subdomains) is then advanced synchronously by $\Delta t^{\text {system }}$. The advantages of this scheme over the static-synchronous scheme implemented above are:

1. improvements in solution accuracy for all subdomains for all time steps;

2. improved description of interfacial dynamics and transients; and

3. possibly more efficient time stepping, as system time steps can increase or decrease as appropriate in response to system equilibria and transients.

The essential steps in the dynamic-synchronous method are summarised in Table 4. Improved time stepping methods are currently under investigation by the authors. 
TABLE 4: Dynamic-synchronous interfacial time stepping scheme.

1 determine the set $\left\{\Delta t_{\max }^{i}\right\}, i=1, \ldots, N$

$\Delta t^{\text {system }}=\min \left(\left\{\Delta t_{\max }^{i}\right\}\right)$

advance all operators $H^{i}$ by $\Delta t^{\text {system }}$

$t^{\text {system }}=t^{\text {system }}+\Delta t^{\text {system }}$

updateinterfacial conditions using $H^{i}$ solutions

goto 1

\section{Application-hydrocarbon biodegradation in a capped soil column}

As an example of the use of the numerical implementation of the interface conditions, the problem of multispecies vapour transport in a variably saturated $3 \mathrm{~m}$ soil column is examined. This one-dimensional problem is complicated by the presence of a thin $(1.5 \mathrm{~mm})$ permeable cap (geomembrane) at the ground surface, thereby dividing the system into four subdomains. In vertical order, from below, these are the saturated (horizontally advecting) groundwater zone, the variably saturated soil column, the permeable capping, and the atmosphere above. Vapour transport in each of the four subdomains is controlled by quite different physical operators. Here, for simplicity, the behaviour of the saturated groundwater zone and the atmospheric zone is parameterised in terms of system boundary conditions to the lower 
boundary of the soil column and to the upper surface of the capping material.

The soil column contains a hydrocarbon phase, variably distributed in the vertical, that is volatilising into the soil gas, where it diffuses through the soil column. The hydrocarbon vapour may dissolve into soil water, where it may be consumed by aerobic microbial action. The rate of microbial degradation may be modelled by a Monod kinetic term, directly coupling local hydrocarbon and oxygen concentrations. Simple Fickian diffusion is assumed to apply in the permeable capping. With various assumptions regarding soil continua, local gas-water equilibria etc, and following the detailed discussion in Öhman [9], the relevant operators, $H^{1}$ (soil column) and $H^{2}$ (cap), can be shown to assume the forms:

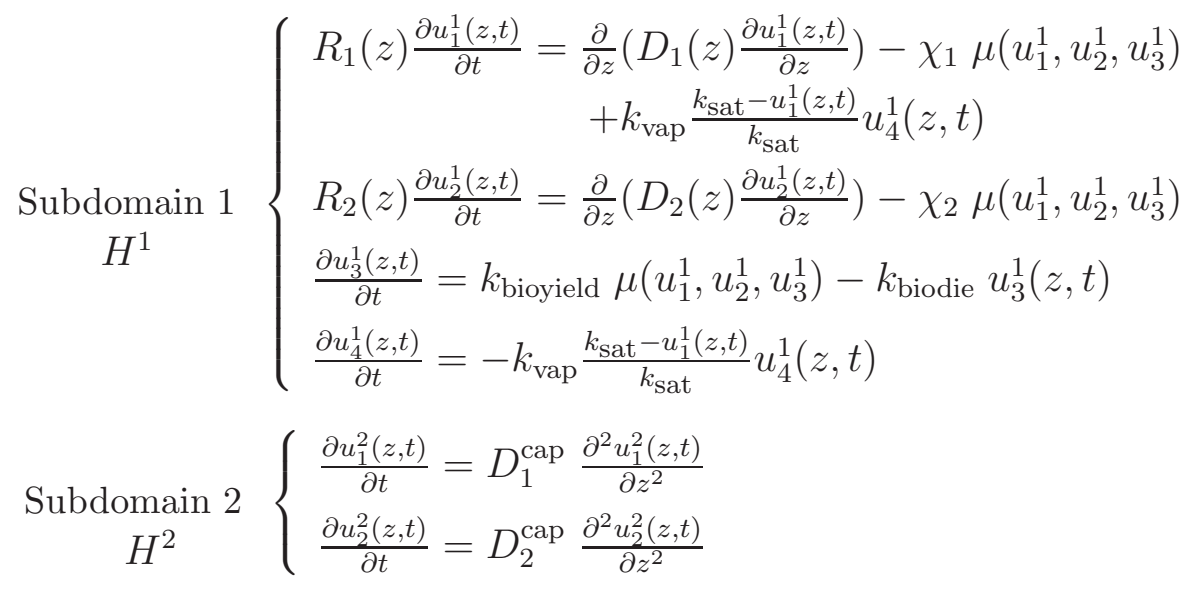

where the $k$ quantities are constants, the $\chi$ parameters indicate stoichiometric relations appropriate for the microbial degradation of the hydrocarbon, and 
the microbial degradation term $\mu$ is given by the coupled form

$$
\mu\left(u_{1}^{1}, u_{1}^{1}, u_{1}^{1}\right)=\sigma_{1} \frac{u_{1}^{1}}{u_{1}^{1}+\sigma_{2}} \frac{u_{2}^{1}}{u_{2}^{1}+\sigma_{3}} \frac{u_{3}^{1}}{u_{3}^{1}+\sigma_{4}}
$$

In this simplified model, components 1, 2 and 3 in subdomain 1 are identified with the hydrocarbon vapour phase, the oxygen gas phase and the microbial population, respectively. Component 4 represents an immobile non-aqueous phase liquid (NAPL) distribution that volatilises, supplementing the hydrocarbon vapour phase. The $R(z)$ and $D(z)$ functions are retardation and effective diffusion coefficients, respectively. These coefficients reflect variations in gas/water phase tortuosities and volumetric measures, and are complicated functions of elevation in the soil column. In subdomain 2, a simple uncoupled twin-component model is used to describe the diffusion of the hydrocarbon vapour and oxygen in the capping medium. The soil-capping interface generates partitioning discontinuities in the mobile (hydrocarbon and oxygen) phases. For polymeric capping, appropriate $\alpha$ values for the soil gas-capping interface range from 0.01 to 0.0001 .

Figure 2 shows results for the example problem above, using hydrocarbon parameters appropriate for benzene, highlighting the non-linear nature of the solutions in subdomain 1 , and the significant change of scale and interfacial mass transfer mechanisms for the hydrocarbon in subdomain 2 . 


\section{Application-hydrocarbon biodegradation in a capped soil column C1461}
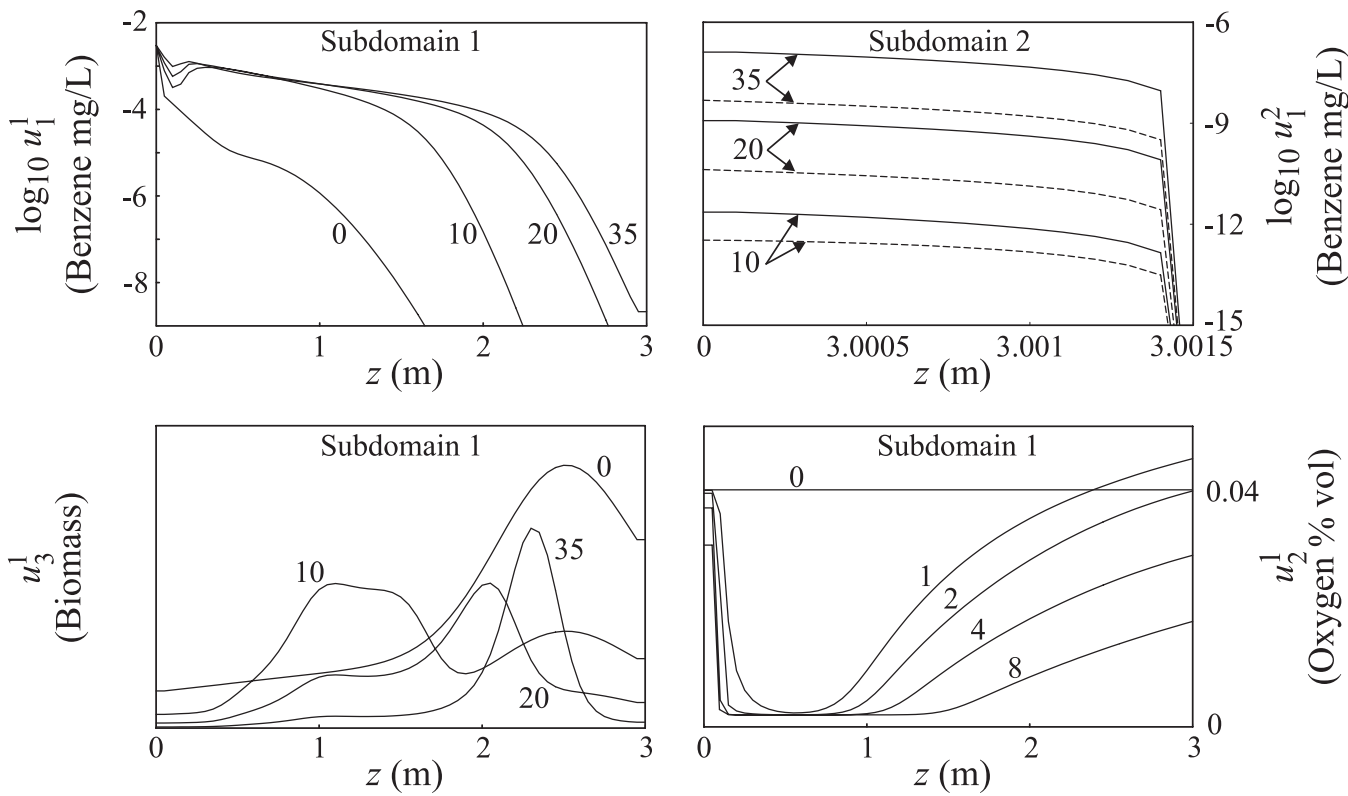

Figure 2: Solution components for the soil contamination example. Numbers indicate solution times (days). The top right plot shows capping concentrations determined using either an instantaneous (solid line) or rate-limited (dashed line) interfacial condition. 


\section{Conclusions}

Solving multiphysics problems in multidomain systems hinges on the development of stable interfacial time stepping algorithms. These algorithms advance subdomain solutions in time in parallel, mediating information flow through the system. A simple synchronous algorithm, used in conjunction with a flexible solver code, yields reasonable results for complicated chemical transport applications. However, acceptable solution convergence is accompanied by fine spatial-temporal grids, indicating the need for more efficient interfacial time stepping algorithms.

Acknowledgements: The authors gratefully acknowledge J. T. Bongiovanni (Physics Department, University of Western Australia) for helpful discussions. This work was part supported by BP Oil Australia.

\section{References}

[1] M. Y. Corapcioglu and A. L. Baehr. A compositional multiphase model for groundwater contamination by petroleum products: 1 . Theoretical considerations. Water Resour. Res., 23(1):191-200, 1987. C1447

[2] M. G. Trefry. SMF2D - A general continuum code for simulating transport in stratified porous media: Development and validation. In 
C. D. Johnston, editor, Contaminated Site Remediation: Challenges posed by urban and industrial contamination, Proceedings of the 1999 Contaminated Site Remediation Conference, Fremantle, pages 275-281. Centre for Groundwater Studies, CSIRO Land and Water, Wembley, Western Australia, 1999. C1449, C1454

[3] M. G. Trefry and D. S. Whyte. Analytical solutions for partitioned diffusion in laminates: I. Initial value problem with steady Cauchy conditions. Transport Porous Med., 37(1):93-128, 1999. C1449, C1454, C1454

[4] J. G. Blom, R. A. Trompert, and J. G. Verwer. Algorithm 758: VLUGR2: a vectorizable adaptive grid solver for PDEs in 2D. ACM T. Math. Software, 22(3):303-328, 1996. C1450

[5] T. P. Mathew, P. L. Polyakov, G. Russo, and J. Wang. Domain decomposition operator splittings for the solution of parabolic equations. SIAM J. Sci. Comput., 19(3):912-932, 1998. C1451

[6] D. Amitai, A. Averbuch, M. Israeli, and S. Itzikowitz. Implicit-explicit parallel asynchronous solver of parabolic PDEs. SIAM J. Sci. Comput., 19(4):1366-1404, 1998. C1451

[7] M. G. Trefry. Analytical solutions for partitioned diffusion in laminates: II. Harmonic forcing conditions. Transport Porous Med., 37(2):183-212, 1999. C1454 
[8] M. G. Trefry, J. Öhman, and G. B. Davis. A simple numerical approach for assessing multiphysics processes in partitioning systems. Applied Math. Modelling, accepted, 2000. C1454

[9] J. Öhman. Modelling transport and degradation dynamics of BTEX vapours in a contaminated vadose zone. Master's thesis, Uppsala University School of Engineering, Uppsala, Sweden, 1999. C1459 